\title{
Multifocal Cellulitis due to Disseminated Neisseria Gonorrhoeae in a Male Patient
}

\author{
Yusuke Yoshino ${ }^{\mathrm{a}, \mathrm{b}}$, Minami Abe ${ }^{\mathrm{a}}$, Kazunori Seo ${ }^{\mathrm{a}}$, Ichiro Koga ${ }^{\mathrm{a}}$, \\ Takatoshi Kitazawa ${ }^{a}$, Yasuo Ota ${ }^{\mathrm{a}}$
}

\begin{abstract}
We report a rare case of disseminated gonococcal infection in a 37 -year-old man presenting with multifocal cellulitis. The patient presented with fever and painful swelling of the right foot and left hand, and was admitted to our hospital. CT scanning of the extremities revealed multifocal cellulitis. Transthoracic echocardiography findings were normal, and piperacilin/tazoactam therapy was initiated. On antibiotic day 4, Neisseria gonorrhoeae was cultured from a purulent effusion collected from a focal site. Chlamydia trachomatis was detected in urine samples by PCR. We made the diagnosis of multifocal cellulitis due to $N$. gonorrhoeae in a patient with chlamydia urethritis. The antibiotic agent was changed from piperacilin/tazobactam to ceftriaxone. Levofloxacin was also administered for chlamydia urethritis. By admission day 14, all lesions had resolved and administration of antibiotic agents was terminated. Disseminated gonococcal infection, although rare, should be included in the differential diagnosis of all sexually active patients who present with multifocal cellulitis - also a rare condition, particularly in light of the fact that in recent times, patterns of sexual activity have changed, which was a pertinent factor in this case.
\end{abstract}

Keyword: Neisseria gonorrhoeae; Disseminated gonococcal infection; Cellulitis

\section{Introduction}

Neisseria gonorrhoeae are gram-negative cocci that can cause sexually transmitted disease, including urethritis, epi-

\footnotetext{
Manuscript accepted for publication January 3, 2014

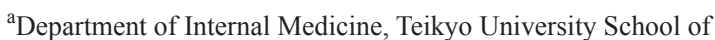
Medicine, 2-11-1 Kaga, Itabashi-ku, Tokyo 173-8606, Japan

${ }^{\mathrm{b}}$ Corresponding author: Yusuke Yoshino, Department of Internal Medicine, Teikyo University School of Medicine, 2-11-1 Kaga, Itabashi-ku, Tokyo 173-8606, Japan.

Email: yyoshino@med.teikyo-u.ac.jp

doi: http://dx.doi.org/10.14740/jocmr1732w
}

didymitis or cervicitis. The usual symptoms of focal infection in men are burning on urination and penile discharge. Women, on the other hand, are asymptomatic half the time, or have vaginal discharge and pelvic pain.

$N$. gonorrhoeae also can cause systemic infection. At first, $N$. gonorrhoeae infects locally. Subsequently, it increases in the focal site, and then spreads systemically. Disseminated gonococcal infection (DGI) results from bacteremic spread of $N$. gonorrhoeae, which can lead to a variety of clinical symptoms and signs. The classical triad of features consists of dermatitis, tenosynovitis and migratory polyarthritis [1]. Endocarditis and meningitis also can occur, but these are extremely rare and have been reported only as case reports. DGI is quite rare, occurring in 0.5 to $3 \%$ of cases [2]. Gender is an important risk factor, in that DGI is four-fold more common among women than men because local symptoms in males are more severe than those in females, and so men tend to be more aware of their infection [3]. Here, we report a rare case of DGI in 37-year-old man presenting as multifocal cellulitis.

\section{Case Report}

The patient presented complaining of fever, throat pain and painful swelling of the right foot and left hand for 1 week, and was admitted to our hospital. Past medical history included a diagnosis of liver cirrhosis (LC) due to alcoholic fatty liver at the age of 36; the patient has continued to drink. He often used the services of sex workers and had a history of gonococcal urethritis at the age of 34 . One month prior to admission, he had unprotected sexual contact. On admission, physical examination revealed a temperature of 39.0 ${ }^{\circ} \mathrm{C}$, pulse rate $124 \mathrm{bpm}$ and blood pressure $140 / 76 \mathrm{mmHg}$. There were painful, reddish and swollen areas on the top of the right foot and the back of the left hand. On the right foot, purulent effusion that was leaking from a small scar was collected for culturing. Blood was also collected for culturing. Admission laboratory findings included a white blood cell count of $13,500 / \mu \mathrm{L}$ with a left shift and a C-reactive protein level of $27.80 \mathrm{mg} / \mathrm{dL}$. A CT scan of the extremities revealed cellulitis in the right foot and left hand (Fig. 1). 


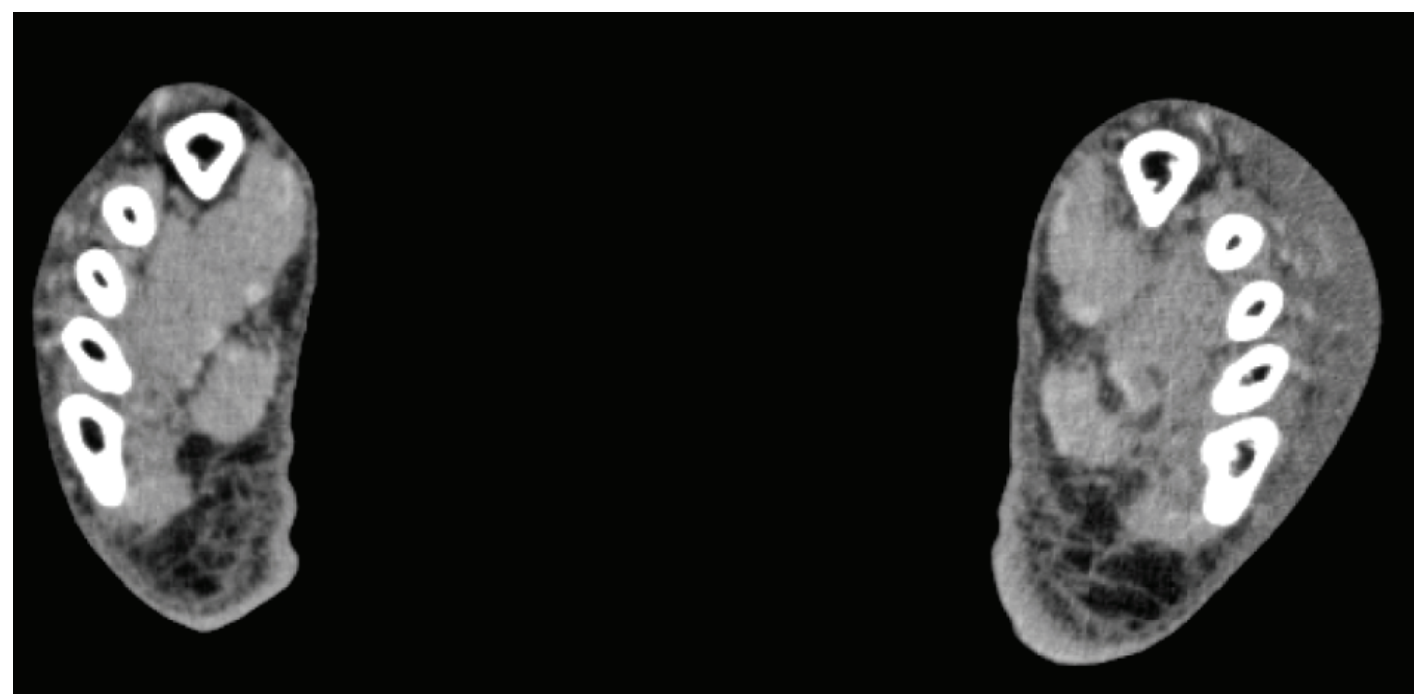

Figure 1. The CT scan of the lower extremities revealed edematous changes in adipose tissue of the upper side of the left foot.

Multifocal cellulitis was diagnosed and piperacilin/tazoactam $(4.5 \mathrm{~g}$ every $6 \mathrm{~h})$ therapy was initiated. Transthoracic echocardiography was performed to search for the focus of this infection, but there were no abnormal findings. On admission day 4, the purulent effusion cultures revealed an $N$. gonorrhoeae strain sensitive to third-generation cephalosporins. We made the diagnosis of multifocal cellulitis due to $N$. gonorrhoeae, and we considered it to be a manifestation of DGI. Piperacilin/tazobactam was changed to ceftriaxone (2 g every $24 \mathrm{~h}$ ). A throat swab obtained 4 days after initiation of antibiotic therapy remained negative for $N$. gonorrhoeae. On admission day 9, Chlamydia trachomatis was detected in urine samples by PCR, and oral levofloxacin $(500 \mathrm{mg} \mathrm{ev}$ ery $24 \mathrm{~h}$ ) was begun. After initiation of antibiotic agents, all symptoms, including the fever and painful swelling of the extremities, gradually declined. After switching antibiotic agents, symptoms continued to decline. On admission day 14 , all symptoms had resolved and administration of antibiotic agents was terminated.

\section{Discussion}

According to a few previous reports, the incidence of DGI has recently increased $[4,5]$, particularly among males, partially because changes in sexual behavior have resulted in the fact that sites other than the genital organs, such as the pharynx, can become reservoirs for N. gonorrhoeae [5]. In our case, although $N$. gonorrhoeae was not detected from the throat swab collected 4 days after initiation of antibiotic therapy, the pharynx might have been harboring the bacteria, because throat pain was reported one week before admission. Therefore, this case might illustrate typical findings given new trends discussed in recent reports.
In our case, $N$. gonorrhoeae was cultured from the purulent effusion, but not from other sites. $N$. gonorrhoeae is difficult to culture due to its fragility. Our diagnosis of DGI was made because $N$. gonorrhoeae was cultured from focal sites and the patient responded to antibiotic treatment, with resolution of all symptoms. Molecular biological testing, including nucleic acid amplification testing, should be performed on samples from patients suspected to have $N$. gonorrhoeae infection who have negative culture results.

Skin and soft tissue infections are unusual presentations of DGI, but there have been several case reports since 1926 [6-8]; although these might be overlooked as rare presentations of DGI, there may be an increase in cases with diverse presenting symptoms in the future because of changes in patterns of sexual activity. For example, it was already reported that an increase in the number of men having sex with men is related to the increase of rectal gonoccocal infection [9]. In addition, the number of immunocompromised patients (like our case, with chronic liver disease) is increasing because of treatment improvements, a fact that also may increase the incidence of DGI and lead to changes in presenting symptoms.

In conclusion, we have reported a rare case of DGI in a male patient having multifocal cellulitis as the presenting symptom. Although definitely rare, cases of DGI presenting with various symptoms will be increasing in the future, perhaps especially in the male population. Our case illustrates the fact that DGI should be included in the differential diagnosis for all sexually active patients who present with multifocal cellulitis.

\section{Conflict of Interest}

The authors of this manuscript have nothing to declare. 


\section{References}

1. O'Brien JP, Goldenberg DL, Rice PA. Disseminated gonococcal infection: a prospective analysis of 49 patients and a review of pathophysiology and immune mechanisms. Medicine (Baltimore). 1983;62(6):395406.

2. Workowski KA, Berman S. Sexually transmitted diseases treatment guidelines, 2010. MMWR Recomm Rep. 2010;59(RR-12):1-110.

3. Wise CM, Morris CR, Wasilauskas BL, Salzer WL. Gonococcal arthritis in an era of increasing penicillin resistance. Presentations and outcomes in 41 recent cases (1985-1991). Arch Intern Med. 1994;154(23):26902695.

4. Update to CDC's Sexually transmitted diseases treatment guidelines, 2010: oral cephalosporins no longer a recommended treatment for gonococcal infections.
MMWR Morb Mortal Wkly Rep. 2012;61(31):590-594.

5. Belkacem A, Caumes E, Ouanich J, Jarlier V, Dellion S, Cazenave B, Goursaud R, et al. Changing patterns of disseminated gonococcal infection in France: cross-sectional data 2009-2011. Sex Transm Infect. 2013;89(8):613-615.

6. Newburger B. Metastatic Intramuscular Gonococcal Abscess. Ann Surg. 1926;84(6):879-885.

7. Jitmuang A, Boonyasiri A, Keurueangkul N, Leelaporn A, Leelarasamee A. Gonococcal subcutaneous abscess and pyomyositis: a case report. Case Rep Infect Dis. 2012;2012:790478.

8. Ho YM, Sebastin SJ, Lim AY. Culture-negative hand abscesses in immunocompetent individuals. Singapore Med J. 2012;53(2):e38-39.

9. Kim AA, Kent CK, Klausner JD. Risk factors for rectal gonococcal infection amidst resurgence in HIV transmission. Sex Transm Dis. 2003;30(11):813-817. 